\title{
miR-532-5p promotes breast cancer proliferation and migration by targeting RERG
}

\author{
LEI HUANG, XIAOQIAO TANG, XIANBIAO SHI and LEI SU \\ Department of Breast and Thyroid Surgery, The Affiliated Drum Tower Hospital, \\ Medical School of Nanjing University, Nanjing, Jiangsu 210008, P.R. China
}

Received August 27, 2018; Accepted October 4, 2019

DOI: $10.3892 /$ etm.2019.8186

\begin{abstract}
Aberrant expression of microRNAs (miRNAs/miRs) mediates the initiation and progression of breast cancer. Therefore, it is important to investigate the molecular mechanisms of miRNAs and their effects on breast cancer progression. In the present study, miR-532-5p was highly expressed in breast cancer tissues compared with normal tissues. In addition, expression of ras-related and estrogen-regulated growth inhibitor (RERG), a tumor suppressor in breast cancer, was negatively correlated with miR-532-5p expression. Inhibition of miR-532-5p significantly elevated RERG at both mRNA and protein levels and inactivated the mitogen-activated protein kinase (MAPK)/ERK signaling pathway. Overexpression of miR-532-5p decreased RERG expression and activated the MAPK/ERK signaling in breast cancer cell line MDA-MB-231. Bioinformatic analysis indicated that RERG 3'-untraslated region contained a putative binding site for miR-532-5p. Dual luciferase assay further validated RERG as a target gene of miR-532-5p. Notably, downregulation of miR-532-5p inhibited MDA-MB-231 cell proliferation and migration, which was partially attenuated upon RERG knockdown. In conclusion, the current study revealed an oncogenic role of miR-532-5p in breast cancer cells via direct targeting of RERG expression.
\end{abstract}

\section{Introduction}

Breast cancer is the most commonly diagnosed cancer type in women, affecting approximately $10 \%$ of women during their lifetime (1). Breast cancer is a heterogeneous disease which is classified into several major subtypes according to the expression of the estrogen receptor (ER), progesterone receptor (PR) and human epidermal growth factor receptor (HER2/neu) (2). Targeted therapies, such as tamoxifen and trastuzumab, have

Correspondence to: Dr Lei Su, Department of Breast and Thyroid Surgery, The Affiliated Drum Tower Hospital, Medical School of Nanjing University, 321 Zhongshan Road, Nanjing, Jiangsu 210008, P.R. China

E-mail: suleinju@sohu.com

Key words: microRNA-532-5p, ras-related and estrogen-regulated growth inhibitor, breast cancer improved the outcomes of patients with $\mathrm{ER}^{+}$or $\mathrm{HER} 2^{+}$breast cancer $(3,4)$. However, a number of patients with breast cancer exhibit de novo or acquired target therapy resistance $(5,6)$. In addition, chemotherapy is the only treatment option for patients with triple-negative breast cancer (TNBC; ER-/HER2//PR') and targets for the effective treatment remain to be elucidated (7). Therefore, the molecular mechanism of breast cancer progression requires further research to fulfill clinical needs.

Previous studies investigated the role of non-coding RNAs in cancer progression and their application as biomarkers and targets for the treatment of patients with cancer $(8,9)$. MicroRNAs (miRNAs/miRs) are small, single-stranded, non-coding RNAs (10). Mechanistically, miRNAs bind to complementary binding sites on the 3 '-untranslated regions (3'-UTRs) of target gene mRNAs, resulting in mRNA degradation or inhibition of translation (11). Dysregulation of miRNAs leads to aberrant expression of numerous genes, which is associated with the initiation and development of human diseases, including cancer (12). Differentially expressed miRNAs have been shown to be associated with different molecular subtypes of breast cancer (13). Several miRNAs, whose expression is associated with the clinical outcomes of breast cancer, serve as oncogenes or tumor suppressors in breast cancer (14-16). Recently, miR-532-5p was demonstrated to be significantly upregulated in TNBC tissues compared with normal breast tissues (17). However, whether and how miR-532-5p contributes to the progression of breast cancer remains unknown.

Ras-related and estrogen-regulated growth inhibitor (RERG) was first identified using microarray analysis in 2001 (18). Decreased expression of RERG is observed in breast tumor tissues and is associated with poor clinical prognosis in breast cancer (18). In breast cancer cells, overexpression of RERG inactivated the Ras/mitogen-activated protein kinase (MAPK)/ERK signaling, leading to the inhibition of cell proliferation, migration and invasion (19).

In the present study, compared with the normal tissues, miR-532-5p was significantly upregulated in breast cancer tissues. Overexpression of miR-532-5p reduced the expression of RERG at both mRNA and protein levels and activated MAPK/ERK signaling in breast cancer cells. In contrast, downregulation of miR-532-5p expression inhibited MDA-MB-231 cell proliferation and migration, which was partially reversed by RERG knockdown. These findings suggest an oncogenic role of miR-532-5p in breast cancer. 


\section{Materials and methods}

Patient samples. Tumor tissues and matched normal tissues were collected from 20 female patients with breast cancer (age range, 25-65 years) between June 2014 and September 2017 at the Affiliated Drum Tower Hospital of Nanjing University (Nanjing, China). Written consent was obtained from all participants prior to sample collection. Ethical approval was received from the Ethic Committee of Nanjing University before initiation of the current study. All experimental procedures were conducted under the supervision of the Ethic Committee of Nanjing University. The tissues were immediately stored in a $-80^{\circ} \mathrm{C}$ refrigerator upon surgical removal before subjecting to the following experiments.

Cell culture. TNBC cell lines (BT549, HS578T, MDA-MB-231) and a normal epithelial breast cell line MCF10A were purchased from American Type Culture Collection and used during the first six months after purchase. MCF10A cells were cultured in Mammary Epithelial Cell Growth Medium (Lonza Group, Ltd.) containing $100 \mathrm{ng} / \mathrm{ml}$ cholera toxin (Sigma-Aldrich; Merck KGaA). BT549, HS578T and MDA-MB-231 cells were maintained in DMEM supplemented with 10\% FBS (Gibco; Thermo Fisher Scientific, Inc.). All cells were cultured in a humidified incubator at $37^{\circ} \mathrm{C}$ with $5 \% \mathrm{CO}_{2}$.

Overexpression and inhibition of $m i R-532-5 p$. miR-negative control (NC) inhibitor, miR-532-5p inhibitor, miR-NC mimic and miR-532-5p mimic were purchased from Suzhou GenePharma Co., Ltd. miR-NC inhibitor and miR-NC mimic served as negative controls for miR-532-5p inhibitor and miR-532-5p mimic, respectively. For the manipulation of miR-532-5p, $50 \mathrm{nM}$ miR-532-5p mimic, miR-532-5p inhibitor, miR-NC mimic or miR-NC inhibitor was transfected into MDA-MB-231 cells using Lipofectamine $3000^{\circledR}$ (Invitrogen; Thermo Fisher Scientific, Inc.) according to the manufacturer's protocol. After $48 \mathrm{~h}$, the RNA and protein were extracted from cells and subjected to the subsequent experiments. The following sequences were used: miR-NC inhibitor, 5'-UUC UCCGAACGUGUCACGU-3'; miR-532-5p inhibitor, 5'-ACG GUCCUACACUCAAGGCAUG-3'; miR-NC mimic, 5'-AUU GGAACGAUACAGAGAAGA-3'; and miR-532-5p mimic, 5'-CAUGCCUUGAGUGUAGGACCGU-3'.

Knockdown of RERG. Control siRNA and RERG siRNA were purchased from Suzhou GenePharma Co., Ltd. For the knockdown of RERG, 50 nM RERG siRNA or control siRNA was transfected into MDA-MB-231 cells using Lipofectamine RNAiMax (Invitrogen; Thermo Fisher Scientific, Inc.) according to the manufacturer's protocol. After $48 \mathrm{~h}$, the RNA and protein were extracted from cells and subjected to the subsequent experiments. The following sequences were used: Control siRNA, 5'-UAAGGCUAUGAAGAGAUAC-3'; RERG siRNA, 5'-CAUCUAGGAUGUUCUUAAGUGGC-3'.

Cell proliferation assay. Cell proliferation was evaluated using a Cell Counting Kit-8 (CCK-8; Dojindo Molecular Technologies, Inc.) following the manufacturer's protocol. In brief, 1,000 MDA-MB-231 cells/well were seeded into 96-well plates. On the next day, the aforementioned miR-NC inhibitor or miR-532-5p inhibitor (50 $\mathrm{nM}$ ) and control siRNA or RERG siRNA $(50 \mathrm{nM})$ were co-transfected into MDA-MB-231 cells using Lipofectamine ${ }^{\circledR}$ RNAiMAX (Thermo Fisher Scientific, Inc.). After $8 \mathrm{~h}$, the medium containing transfection reagents was replaced with fresh DMEM supplemented with 10\% FBS. At $0,24,48,72$ and 96 h, $10 \mu$ l CCK-8 solution was added into each well and incubated for $2 \mathrm{~h}$. The medium containing the CCK-8 solution was then transferred into another 96-well plate, where absorbance was measured at a wavelength of $450 \mathrm{~nm}$ to reflect the cell number in each group for the calculation of cell proliferation.

Cell migration assay. The cell migration ability was determined using a wound healing assay. Briefly, $1 \times 10^{6}$ MDA-MB-231 cells were seeded in each well of six-well plates and cultured until reaching $90 \%$ confluence. A wound was created at the central area of the cell monolayer by scratching the plate with a $10 \mu \mathrm{l}$ pipette tip. The medium was discarded and the cells were rinsed with PBS twice. Subsequently, DMEM with no serum was added into each well. The images of the migrated area were captured using an inverted light microscope at 0 and $30 \mathrm{~h}$. The percentage migrated area was calculated with Image Pro Plus (Media Cybernetics, Inc.).

RNA isolation and RT-qPCR. Total RNA was isolated from cells and tissues using TRIzol ${ }^{\circledR}$ reagent (Invitrogen; Thermo Fisher Scientific, Inc.) according to the manufacturer's protocol. The RNA concentration was determined using a NanoDrop ${ }^{\mathrm{TM}} 2000$ spectrophotometer (Thermo Fisher Scientific, Inc.). For mRNA quantification, RNA was reverse transcribed into cDNA with a PrimeScript RT Master Mix kit (Takara Bio, Inc.) following the manufacturer's instructions. qPCR was performed using a SYBR Green qPCR Master Mix kit (Takara Bio, Inc.). The stem-loop method was used for detection of miRNA expression (20). For miRNA quantification, RNA was reverse-transcribed using a Mir-X miRNA First Strand Synthesis kit (Takara Bio, Inc.) followed by RT-qPCR with Mir-X ${ }^{\mathrm{TM}}$ miRNA qRT-PCR SYBR kit (Takara Bio, Inc.). The thermocycling conditions for the qPCR was as follows: Initial denaturation at $95^{\circ} \mathrm{C}$ for $30 \mathrm{sec}$, followed by 35 cycles of $95^{\circ} \mathrm{C}$ for $10 \mathrm{sec}$ and $60^{\circ} \mathrm{C}$ for $30 \mathrm{sec}$. GAPDH and U6 served as internal controls for mRNA and miRNA, respectively. The relative expression of mRNA and miRNA was calculated using the $2^{-\Delta \Delta C q}$ method (21). The following primer sequences were used: GAPDH forward, 5'-CCTGCA CCACCAACTGCTTA-3' and reverse, 5'-GGCCATCCACAG TCTTCTGAG-3'; RERG forward, 5'-GAATCAACCTAC CGACACCAAG-3' and reverse, 5'-CTCCCTCTGAATGGT ATCTTCCT-3'; U6 forward, 5'-GCGCGTCGTGAAGCG TTC-3' and reverse 5'-GGTCGGCTTTCAGTCGGATGT T-3'; miR-532-5p forward 5'-TGGGTCCTTGCCTTGAGT GTAG-3' and reverse 5'-GGTCGGCTTTCAGTCGGATGT T-3'; stem-loop-miR-532-5p, 5'-GTCGTATCCAGTGCAGGG TCCGAGGTATTCGCACTGGATACGACACGGTC-3'; and stem-loop-U6, 5'-GTCGTATCCAGTGCAGGGTCCGAG GTATTCGCACTGGATACGACAAATATG-3'.

Protein isolation and western blotting. Proteins were isolated from MDA-MB-231 cells with a RIPA lysis buffer (Sigma-Aldrich; Merck KGaA). The concentration of protein 
A

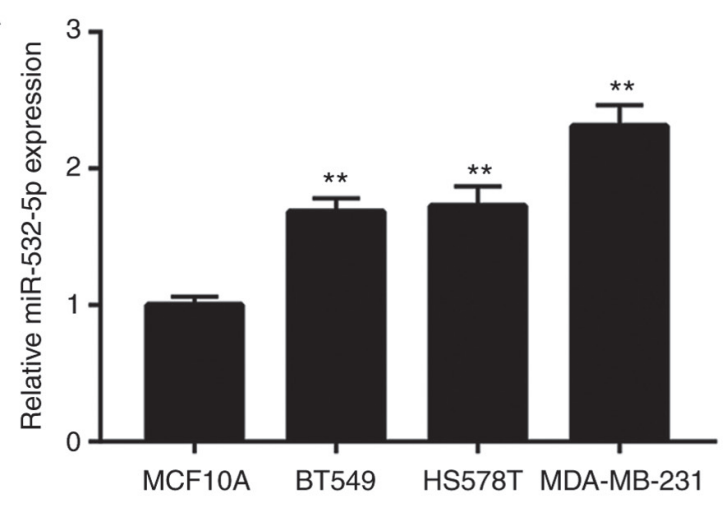

B

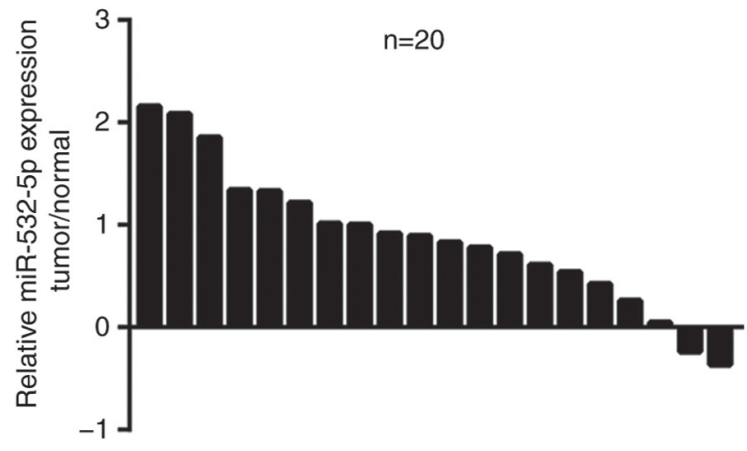

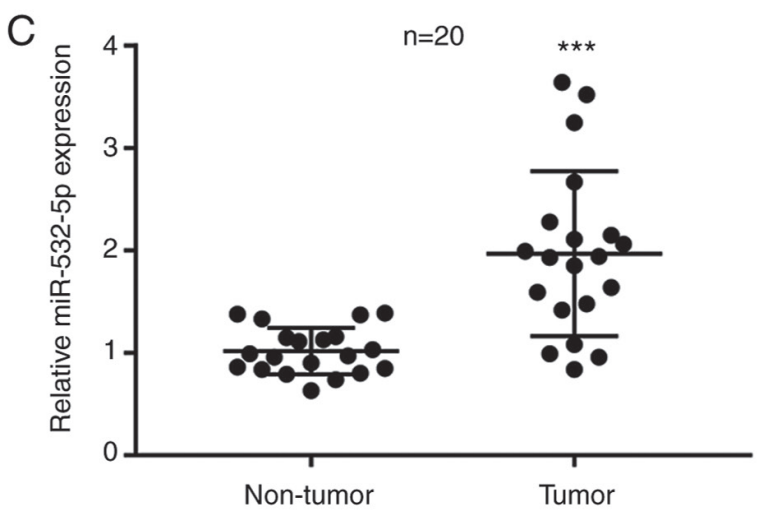

Figure 1. Overexpression of miR-532-5p in breast cancer cells and tumor tissues. (A) Compared with immortalized non-tumorigenic breast epithelial cell line MCF10A, expression of miR-532-5p was increased in TNBC cell lines, including BT549, HS578T, MDA-MB-231. (B) Expression of miR-532-5p was increased in $90 \%$ (18/20) of tumor tissues compared with the adjacent non-tumor tissues. (C) Compared with normal tissues, miR-532-5p expression was significantly increased in breast tumor tissues. ${ }^{* *} \mathrm{P}<0.01$ vs. MCF10A and ${ }^{* * *} \mathrm{P}<0.001$ vs. non-tumor. miR, microRNA.

lysates was determined using a BCA Protein Assay kit (Thermo Fisher Scientific, Inc.). Anti-RERG (cat. no. SAB2107423; 1:2,000) and GAPDH (cat. no. G8795; 1: 5,000) antibodies were purchased from Sigma-Aldrich (Merck KGaA). Anti-dual specificity mitogen-activated protein kinase kinase (MEK; cat. no. 4694; 1:1,000), ERK1/2 (cat. no. 4695; 1:1,000), phosphorylated (p)-MEK (cat. no. 2338; 1:2,000) and p-ERK1/2 (cat. no. 9101; 1:2,000) antibodies were purchased from Cell Signaling Technology, Inc. Horseradish peroxidase-conjugated secondary antibodies against mouse (cat. no. KC-MM-035, 1:10,000) and rabbit (cat. no. KC-RB-035, 1:10,000) were purchased from Aksomics, Inc. For western blotting, $10 \mu \mathrm{g}$ protein/lane was separated using SDS-PAGE ( $8 \%$ gel), transferred onto a PVDF membrane and blocked in 5\% nonfat milk for $1 \mathrm{~h}$ at room temperature. The PVDF membrane was incubated with the aforementioned primary antibodies overnight at $4^{\circ} \mathrm{C}$. Subsequently, the PVDF membrane was washed and incubated with secondary antibodies for $1 \mathrm{~h}$ at room temperature. The bands were visualized using a Pierce ${ }^{\mathrm{TM}}$ ECL Western Blotting Substrate (Thermo Fisher Scientific, Inc.). Densitometric analysis was performed using the Image J software (version 1.51; National Institute of Health).

Dual-luciferase reporter assay. The 3'-UTR sequence of RERG mRNA was amplified from cDNA of MCF10A cells using Takara Ex Taq ${ }^{\circledR}$ Hot Start polymerase (Takara Bio, Inc.) and ligated into the pGL3-basic plasmid (Promega Corporation). Two point mutations were introduced into the pGL3-RERG
3'-UTR-wild-type (WT) construct using QuickChange Site-Directed Mutagenesis kit (Agilent Technologies, Inc.) to generate pGL3-RERG 3'UTR-mutant (Mut). For the dual luciferase reporter assay, $2 \times 10^{6}$ MDA-MB-231 cells were transfected with pGL3-RERG 3'-UTR-WT or pGL3-RERG 3'-UTR-Mut and miR-532-5p mimic or miR-NC mimic using Lipofectamine ${ }^{\circledR}$ RNAiMAX (Thermo Fisher Scientific, Inc.). Relative luciferase activity was calculated by normalizing to that of Renilla luciferase. After $48 \mathrm{~h}$, the relative luciferase activity of each well was detected using the Dual Luciferase Reporter Assay System (Promega Corporation).

Statistical analysis. Data were analyzed using GraphPad Prism 7.0 (GraphPad Software, Inc.) and are presented as the mean \pm SD. The correlation between miR-532-5p and RERG was analyzed using Pearson's correlation analysis. Differences between normal breast tissues and breast cancer tissues were analyzed by paired Student's t-test, while the differences between two independent groups were analyzed using Student's t-test. Differences between multiple groups were analyzed with one-way ANOVA followed by Newman-Keuls test. $\mathrm{P}<0.05$ was considered to indicate a statistically significant difference. All experiments were repeated three times.

\section{Results}

miR-532-5p expression levels is increased in breast cancer cells and tumor tissues. To investigate the role of miR-532-5p 
A

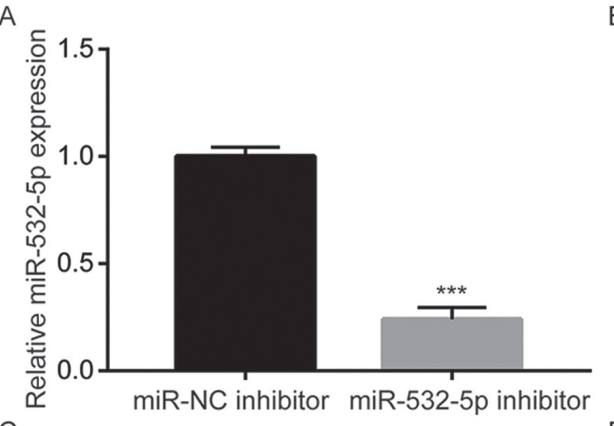

C

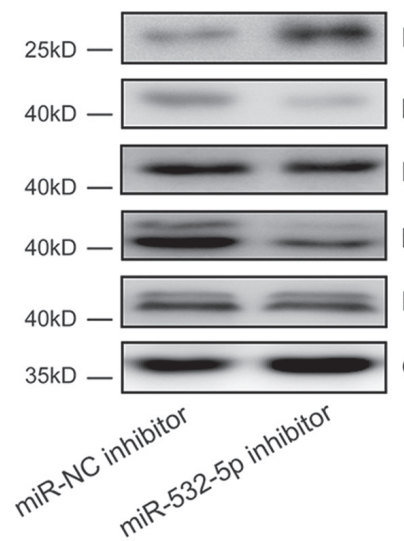

E

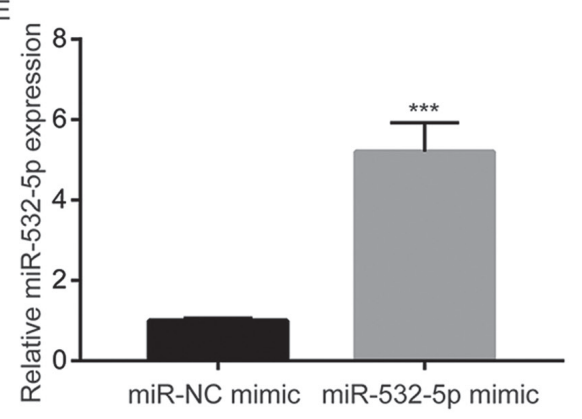

G

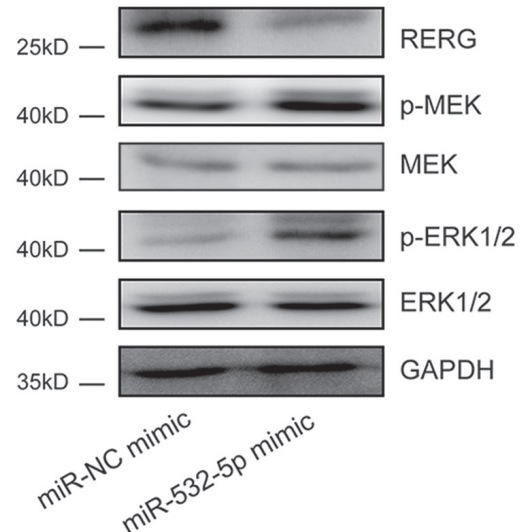

$\mathrm{B}$
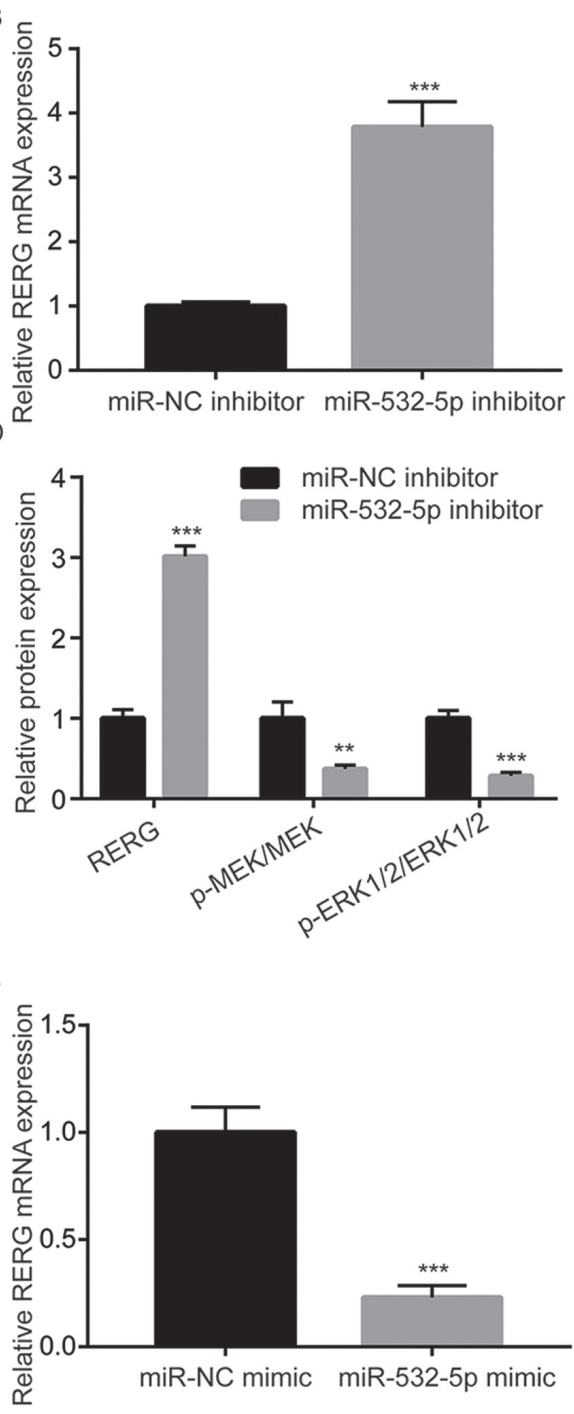

$\mathrm{H}$

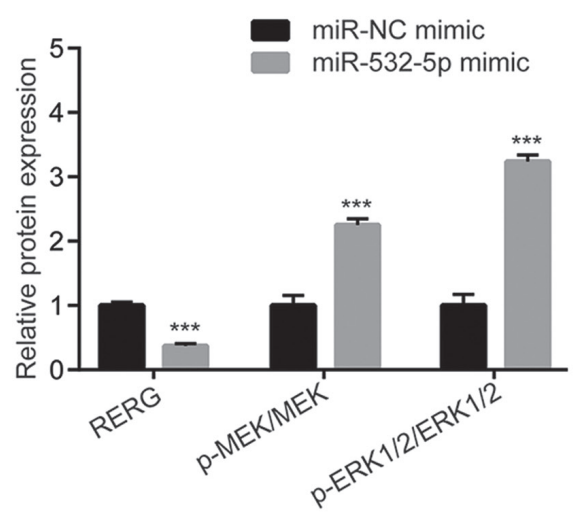

Figure 2. RERG was negatively regulated by miR-532-5p in breast cancer cells. (A) Transfection with miR-532-5p inhibitor decreased miR-532-5p expression in MDA-MB-231 cells. (B) Downregulation of miR-532-5p increased RERG mRNA levels in MDA-MB-231 cells. (C) Downregulation of miR-532-5p increased RERG protein expression levels and decreased p-MET and p-ERK1/2 protein levels in MDA-MB-231 cells. (D) Western blotting results were analyzed quantitatively. (E) Transfection with miR-532-5p mimics successfully increased the expression of miR-532-5p. (F) Upregulation of miR-532-5p decreased RERG mRNA levels in MDA-MB-231 cells. (G) Upregulation of miR-532-5p decreased RERG protein levels and increased p-MET and p-ERK1/2 protein levels in MDA-MB-231 cells. (H) Western blotting results were analyzed quantitatively. ${ }^{* *} \mathrm{P}<0.01$ and ${ }^{* * * *} \mathrm{P}<0.001$ vs. the respective miR-NC group. NC, negative control; miR, microRNA; MEK, dual specificity mitogen-activated protein kinase kinase; RERG, ras-related and estrogen-regulated growth inhibitor.

in breast cancer, RT-qPCR was performed to detect the difference in miR-532-5p expression levels between the immortalized non-tumorigenic breast epithelial cell line MCF10A and TNBC cell lines, including BT549, HS578T and MDA-MB-231. Compared with MCF10A, the expression of miR-532-5p was significantly upregulated in three TNBC cell lines (Fig. 1A). 
Additionally, in 20 pairs of normal breast tissues and breast tumor tissues, miR-532-5p expression was increased in 90\% (18/20) of breast cancer tissues (Fig. 1B). Compared with normal breast tissues, the expression of miR-532-5p was significantly elevated in breast cancer tissues (Fig. 1C). These data indicated that miR-532-5p may be associated with breast cancer.

miR-532-5p inhibits RERG expression and activates the MAPK/ERK pathway in breast cancer cells. Following downregulation of miR-532-5p expression by miR-532-5p inhibitor transfection, RERG mRNA levels were significantly increased (Fig. 2A and B). Furthermore, western blotting results showed that RERG protein levels were also increased upon miR-532-5p downregulation in MDA-MB-231 cells (Fig. 2C and D). In the present study, miR-532-5p downregulation decreased the protein levels of p-MEK and p-ERK1/2 but had no effect on total MEK and ERK1/2 expression in MDA-MB-231 cells (Fig. 2C and D), suggesting inactivation of the MAPK/ERK pathway. However, transfection with miR-532-5p mimic increased miR-532-5p expression levels (Fig. 2E), reduced RERG expression (Fig. 2F) and increased p-MEK/MEK and p-ERK1/2/ERK1/2 ratios in MDA-MB-231 cells (Fig. 2G and H). These findings suggest that miR-532-5p could activate the MAPK/ERK signaling via repression of RERG expression in breast cancer cells.

RERG is a target gene of miR-532-5p in breast cancer cells. To examine whether miR-532-5p directly regulated the expression of RERG in breast cancer cells, bioinformatic analysis was applied to predict the potential binding site between miR-532-5p and the 3'-UTR of RERG mRNA. Sequence alignment showed that there was a putative binding site between these sequences (Fig. 3A). Dual luciferase reporter assays revealed that co-transfection of miR-532-5p mimic and RERG 3'-UTR-WT significantly decreased the luciferase activity while co-transfection of miR-532-5p mimic and RERG 3'-UTR-Mut did not change the luciferase activity in MDA-MB-231 cells, compared with the respective miR-NC mimic groups (Fig. 3B). The data indicated that miR-532-5p directly bound to the 3'-UTR of RERG mRNA, leading to the downregulation of RERG expression.

Downregulation of miR-532-5p inhibits cell proliferation via upregulation of RERG in breast cancer cells. To further study whether miR-532-5p regulated cell proliferation via repression of RERG, cell proliferation ability was detected in MDA-MB-231 cells transfected with miR-532-5p inhibitor with or without RERG siRNA. Transfection of RERG siRNA significantly decreased RERG protein expression in MDA-MB-231 cells (Fig. 4A and B). Transfection of miR-532-5p inhibitor increased RERG protein expression in MDA-MB-231 cells and co-transfection of RERG siRNA decreased RERG protein expression (Fig. 4C and D). Cell proliferation assay showed that downregulation of miR-532-5p significantly inhibited cell proliferation ability, compared with the group transfected with miR-NC inhibitor and control siRNA, which was partially reversed by silencing RERG expression (Fig. 4E), suggesting that miR-532-5p functioned as a negative regulator of cell growth through repression of RERG expression.
A RERG 3'UTR-WT 3'-TTACGGAAACGTTTTTGAAGTTGG-5'
miR-532-5p 5'-CAUGCCUUGAGUGUAGGACCGU-3'
RERG 3'UTR-Mut 3'-TTACCCAAACGTTTTTGAAGTTGG-5'

B
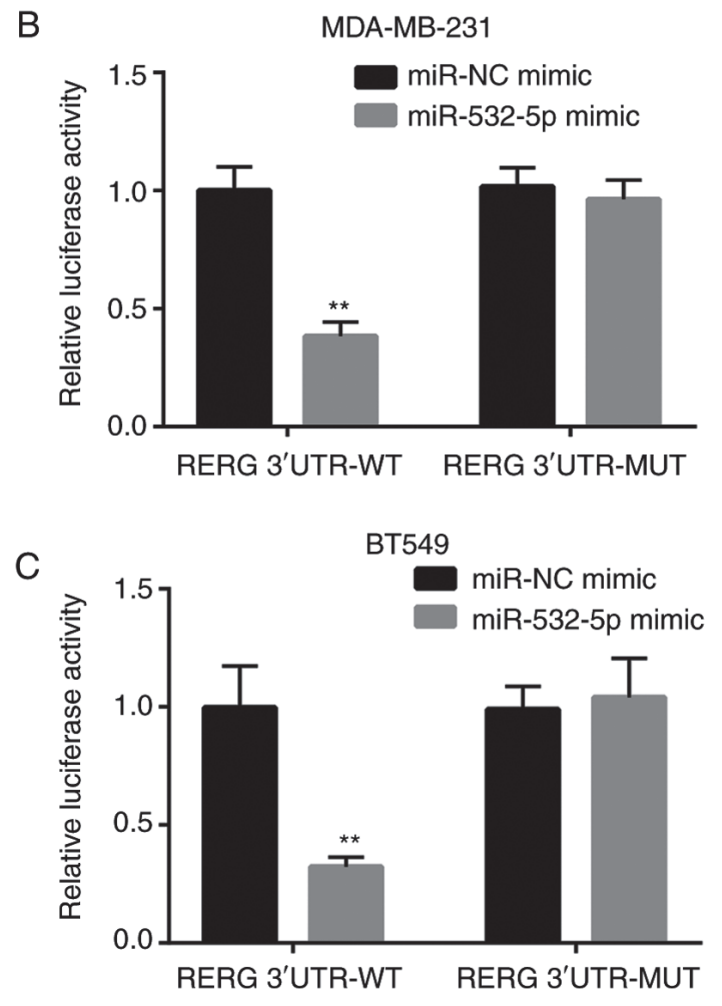

Figure 3. RERG is a target gene of miR-532-5p in breast cancer cells. (A) Sequence alignment showed that there was a putative binding site for miR-532-5p within the 3'-UTR of RERG mRNA. Two point mutations were introduced in the 3'-UTR-WT of RERG to establish RERG 3'-UTR-Mut. (B) In a dual luciferase reporter assay, transfection of miR-532-5p mimic reduced luciferase activity of MDA-MB-231 cells transfected with RERG 3'-UTR-WT. (C) In a dual luciferase reporter assay, transfection of miR-532-5p mimic reduced luciferase activity of BT549 cells transfected with RERG 3'-UTR-WT. ${ }^{* *} \mathrm{P}<0.01$ vs. miR-NC mimic. 3'-UTR, 3'-untranslated region; miR, microRNA; NC, negative control; WT, wild type; Mut, mutant; RERG, ras-related and estrogen-regulated growth inhibitor.

Downregulation of miR-532-5p inhibits cell migration via upregulation of RERG in breast cancer cells. Wound healing assays showed that downregulation of miR-532-5p reduced the number of cells that migrated towards the wound area, compared with the group transfected with miR-NC inhibitor and control siRNA, which was antagonized by RERG silencing (Fig. 5A and B), suggesting that miR-532-5p functioned as a negative regulator of cell migration through repression of RERG expression.

Expression of miR-532-5p is negatively correlated with RERG mRNA expression in breast cancer tissues. The differences of RERG mRNA level were analyzed between breast cancer tissues and normal breast tissues from 20 patients with breast cancer. The results showed that RERG mRNA expression was significantly decreased in breast cancer tissues compared with normal tissues (Fig. 6A). Moreover, Pearson's correlation analysis demonstrated that there was a strong negative correlation 
A

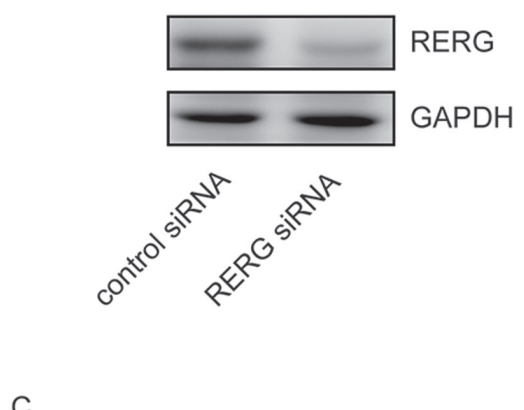

C

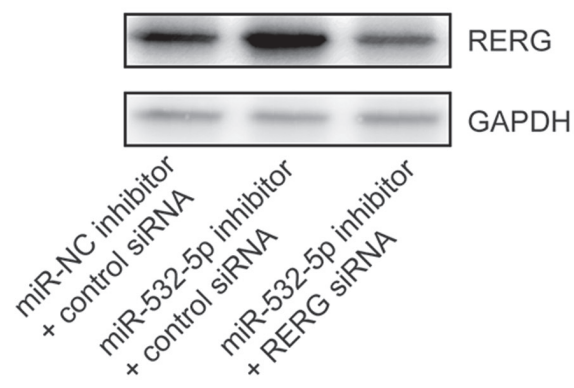

$\mathrm{E}$

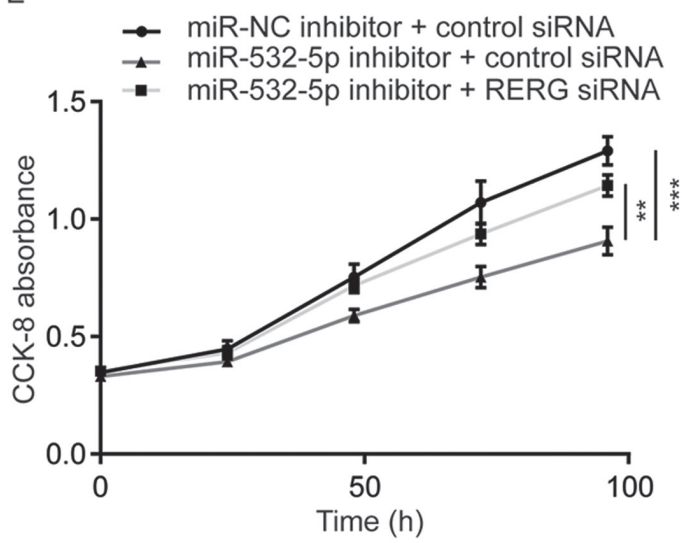

$\mathrm{B}$

D
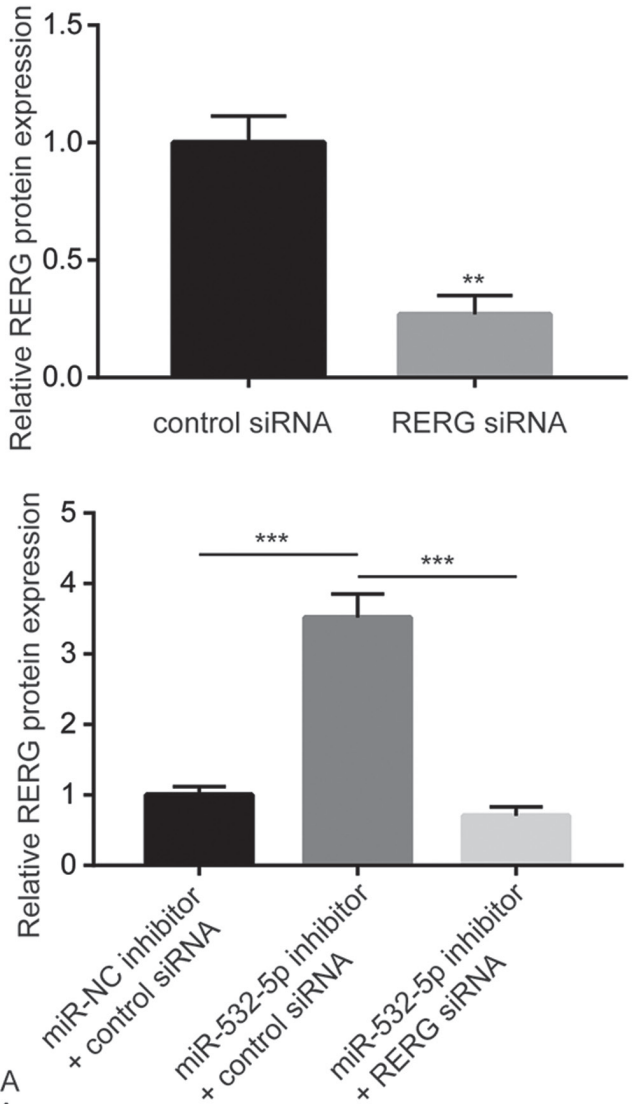

Figure 4. Transfection of miR-532-5p inhibitor inhibits cell proliferation via upregulation of RERG in breast cancer cells. (A) Transfection of RERG siRNA decreased RERG protein expression in MDA-MB-231 cells. (B) Quantification of RERG protein expression. (C) Transfection of miR-532-5p inhibitor increased RERG protein levels, which was partially attenuated by RERG siRNA. (D) Quantification of RERG protein expression. (E) Transfection of miR-532-5p inhibitor inhibited cell proliferation of MDA-MB-231 cells, which was partially rescued by RERG siRNA. ${ }^{* *}$ P $<0.01$ and ${ }^{* * *} \mathrm{P}<0.001$. miR, microRNA; NC, negative control; siRNA, small interfering RNA; RERG, ras-related and estrogen-regulated growth inhibitor; CCK-8, cell counting kit-8.

between miR-532-5p levels and RERG mRNA levels in breast cancer tissues (Fig. 6B).

\section{Discussion}

Dysregulation of miRNAs is a critical step for the initiation, metastasis, angiogenesis, chemoresistance, radiotherapy resistance and maintenance of stemness in breast cancer (22-26). A number of studies identified differentially expressed miRNAs between normal breast tissues and breast cancer tissues $(13,17)$. The following studies validated that these miRNAs were involved in the proliferation and metastasis of breast cancer cells through regulation of their target genes (27-29). Recently, miR-532-5p was revealed as a markedly upregulated miRNA in TNBC (17). The present study investigated the role and mechanism of miR-532-5p in breast cancer cells. Consistently with the results of a previous study (17), the current RT-qPCR results showed that miR-532-5p expression was increased in TNBC cells compared with the immortal breast cells. Downregulation of miR-532-5p decreased cell proliferation and migration ability of MDA-MB-231 cells. These results demonstrated that miR-532-5p may be a new oncogenic miRNA in breast cancer cells.

Sustained activation of the MAPK/ERK signaling promotes proliferation, migration ability and drug resistance 
A
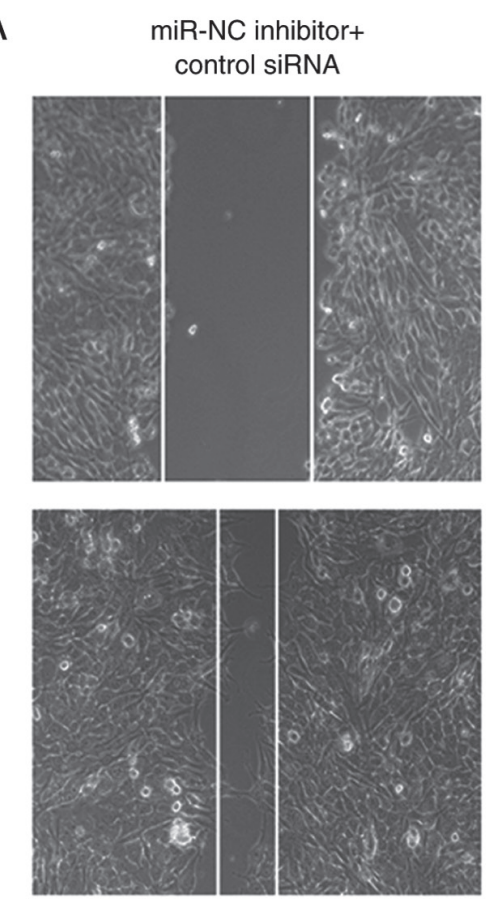

miR-532-5p inhibitor+ control siRNA
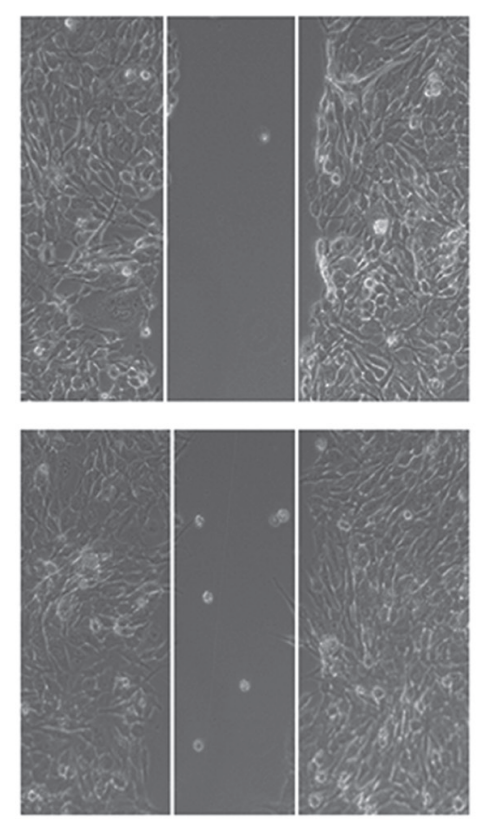

miR-532-5p inhibitor+ RERG SiRNA
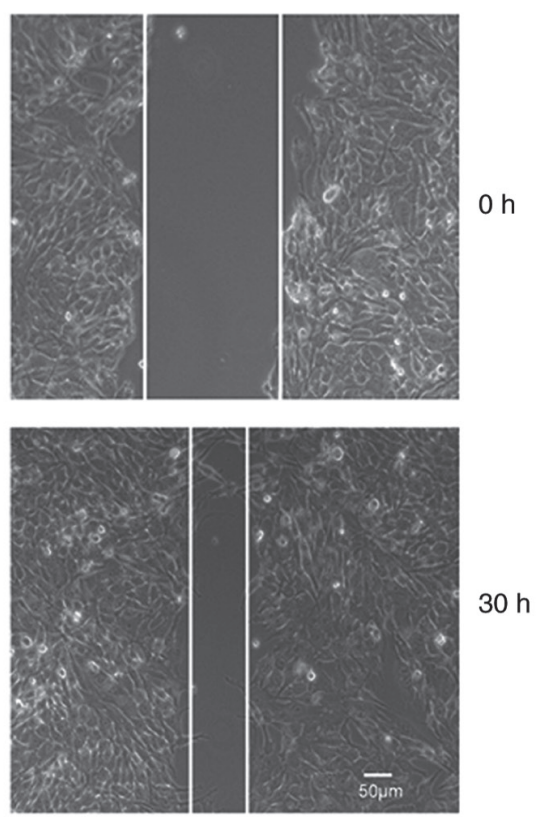

B

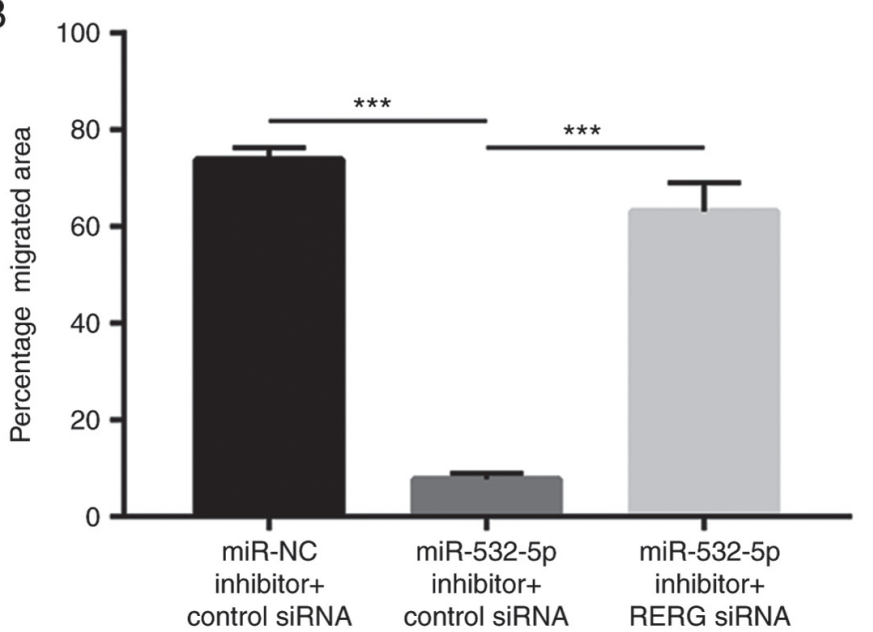

Figure 5. Transfection of miR-532-5p inhibitor inhibits cell migration via upregulation of RERG in breast cancer cells. (A) Transfection of miR-532-5p inhibitor inhibited cell migration of MDA-MB-231 cells, which was partially rescued by RERG siRNA. Magnification, x20. (B) Quantification of migrated area. ${ }^{* * *} \mathrm{P}<0.001$. miR, microRNA; NC, negative control; siRNA, small interfering RNA; RERG, ras-related and estrogen-regulated growth inhibitor.

A

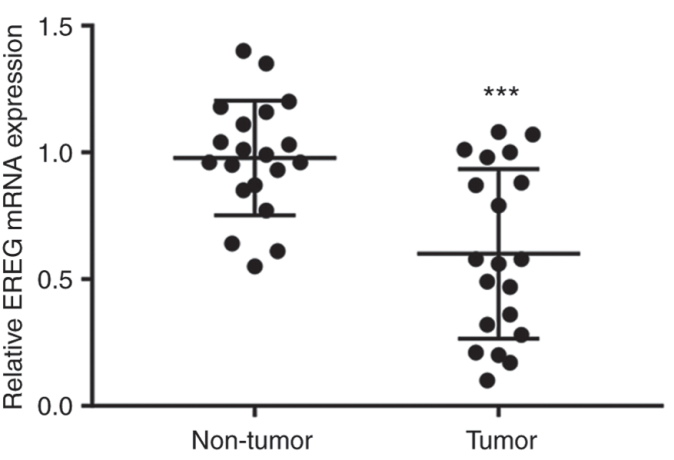

$\mathrm{B}$

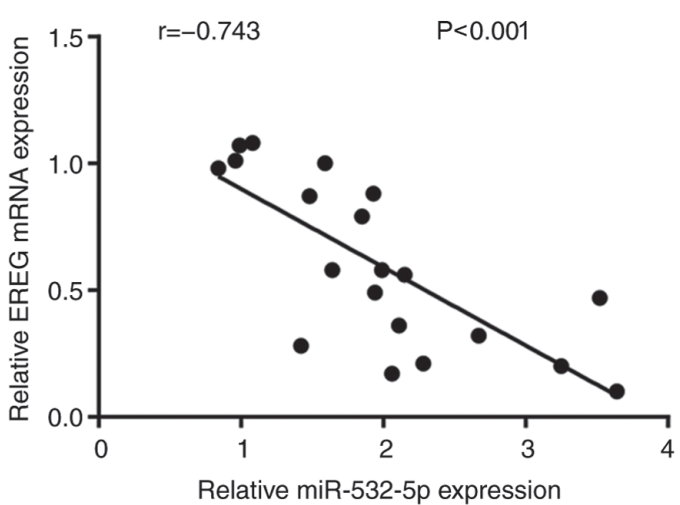

Figure 6. Expression of miR-532-5p is negatively correlated with RERG mRNA levels in breast tumor tissues. (A) Compared with normal tissues, expression level of RERG mRNA was significantly decreased in breast tumor tissues. (B) Pearson's correlation analysis showed that there was a significant negative correlation between miR-532-5p expression and RERG mRNA levels in breast tumor tissues from 20 patients with breast cancer. ${ }^{* * *} \mathrm{P}<0.001$ vs. non-tumor. miR, microRNA; RERG, ras-related and estrogen-regulated growth inhibitor. 
of breast cancer cells (30). RERG was first identified as a ras-like, growth inhibitory protein induced by estrogen in breast cancer cells (18). Subsequent studies revealed that RERG was a negative regulator of multiple oncogenic pathways including the MAPK/ERK signaling in cancer cells (19,31). Furthermore, RERG was also regulated by histone deacetylases and miRNAs $(19,32,33)$. In the current study, miR-532-5p downregulation led to inactivation of the MAPK/ERK signaling in MDA-MB-231 cells. Bioinformatic analysis results indicated that RERG, an upstream negative regulator of the MAPK/ERK signaling pathway, was a potential target gene of miR-532-5p. In addition, downregulation of miR-532-5p also increased RERG mRNA and protein levels in MDA-MB-231 cells. Additionally, the dual luciferase reporter assay validated that RERG was a target gene of miR-532-5p. In several cancer types, including breast cancer, RERG inhibits cell proliferation, migration, invasion and clonogenicity via the MAPK/ERK pathway $(19,31)$. In the present study, cell proliferation and migration assays showed that RERG silencing could reverse miR-532-5p downregulation-induced cell proliferation and migration inhibition. Furthermore, in clinical samples, a significant negative correlation was observed between miR-532-5p expression and RERG mRNA levels. Thus, the current results suggest that miR-532-5p acted as an oncogenic miRNA in breast cancer via repression of RERG. A previous study indicated that in breast cancer cell lines, including MCF7 and Hs578t, RERG was a target gene of miR-382-5p. Via suppression of RERG, miR-382-5p promoted cell growth of breast cancer cells in vitro and in vivo (33). The current and previous results suggest that RERG may be regulated by a complex miRNA network involving miR-382-5p and miR-532-5p in breast cancer cells.

In conclusion, the current study provided new sights into the role of miR-532-5p and the regulatory mechanism of RERG in breast cancer cells. The current study demonstrated that miR-532-5p may be an oncogenic miRNA and may serve as a therapeutic target in breast cancer cells.

\section{Acknowledgements}

Not applicable.

\section{Funding}

The current study was funded by Nanjing Medical Science and Technology Development Project (grant no. YKK17063).

\section{Availability of data and materials}

The datasets used and/or analyzed during the current study are available from the corresponding author on reasonable request.

\section{Authors' contributions}

LH, XT and XS were responsible for clinical sample collection. LS was responsible for study design and supervision, and manuscript preparation, review and editing. Data acquisition and analysis were performed by LH and XT.

\section{Ethics approval and consent to participate}

All patients provided written informed consent before the study and the Ethic Committee of Nanjing University approved the present study.

\section{Patient consent for publication}

Not applicable.

\section{Competing interests}

The authors declare that they have no competing interests.

\section{References}

1. Siegel R, Naishadham D and Jemal A: Cancer statistics, 2013. CA Cancer J Clin 63: 11-30, 2013.

2. McDermott AM, Miller N, Wall D, Martyn LM, Ball G, Sweeney KJ and Kerin MJ: Identification and validation of oncologic miRNA biomarkers for luminal A-like breast cancer. PLoS One 9: e87032, 2014.

3. Notas G, Pelekanou V, Kampa M, Alexakis K, Sfakianakis S, Laliotis A, Askoxilakis J, Tsentelierou E, Tzardi M, Tsapis A and Castanas E: Tamoxifen induces a pluripotency signature in breast cancer cells and human tumors. Mol Oncol 9: 1744-1759, 2015.

4. Ahmed S, Sami A and Xiang J: HER2-directed therapy: Current treatment options for HER2-positive breast cancer. Breast Cancer 22: 101-116, 2015.

5. Karlsson E, Veenstra C, Emin S, Dutta C, Pérez-Tenorio G, Nordenskjöld B, Fornander T and Stål O: Loss of protein tyrosine phosphatase, non-receptor type 2 is associated with activation of $\mathrm{AKT}$ and tamoxifen resistance in breast cancer. Breast Cancer Res Treat 153: 31-40, 2015.

6. Boulbes DR, Chauhan GB, Jin Q, Bartholomeusz C and Esteva FJ: CD44 expression contributes to trastuzumab resistance in HER2-positive breast cancer cells. Breast Cancer Res Treat 151: 501-513, 2015.

7. O'Reilly EA, Gubbins L, Sharma S, Tully R, Guang MH, Weiner-Gorzel K, McCaffrey J, Harrison M, Furlong F, Kell M and McCann A: The fate of chemoresistance in triple negative breast cancer (TNBC). BBA Clin 3: 257-275, 2015.

8. Venkatesh T, Suresh PS and Tsutsumi R: Non-coding RNAs: Functions and applications in endocrine-related cancer. Mol Cell Endocrinol 416: 88-96, 2015.

9. Calore F, Lovat F and Garofalo M: Non-coding RNAs and cancer. Int J Mol Sci 14: 17085-17110, 2013.

10. Yates LA, Norbury CJ and Gilbert RJ: The long and short of microRNA. Cell 153: 516-519, 2013.

11. Bartel DP: MicroRNAs: Genomics, biogenesis, mechanism, and function. Cell 116: 281-297, 2004.

12. Kanwar JR, Mahidhara G and Kanwar RK: MicroRNA in human cancer and chronic inflammatory diseases. Front Biosci (Schol Ed) 2: 1113-1126, 2010.

13. Lowery AJ, Miller N, Devaney A, McNeill RE, Davoren PA, Lemetre C, Benes V, Schmidt S, Blake J, Ball G and Kerin MJ: MicroRNA signatures predict oestrogen receptor, progesterone receptor and HER2/neu receptor status in breast cancer. Breast Cancer Res 11: R27, 2009.

14. Cui S, Liao X, Ye C, Yin X, Liu M, Hong Y, Yu M, Liu Y, Liang $\mathrm{H}$, Zhang $\mathrm{CY}$ and Chen $\mathrm{X}$ : ING5 suppresses breast cancer progression and is regulated by miR-24. Mol Cancer 16: 89, 2017.

15. Shi Y, Luo X, Li P, Tan J, Wang X, Xiang T and Ren G: miR-7-5p suppresses cell proliferation and induces apoptosis of breast cancer cells mainly by targeting REG $\gamma$. Cancer Lett 358: 27-36, 2015.

16. Raychaudhuri M, Bronger H, Buchner T, Kiechle M, Weichert W and Avril S: MicroRNAs miR-7 and miR-340 predict response to neoadjuvant chemotherapy in breast cancer. Breast Cancer Res Treat 162: 511-521, 2017

17. Tsai HP, Huang SF, Li CF, Chien HT and Chen SC: Differential microRNA expression in breast cancer with different onset age. PLoS One 13: e0191195, 2018. 
18. Finlin BS, Gau CL, Murphy GA, Shao H, Kimel T, Seitz RS, Chiu YF, Botstein D, Brown PO, Der CJ, et al: RERG is a novel ras-related, estrogen-regulated and growth-inhibitory gene in breast cancer. J Biol Chem 276: 42259-42267, 2001.

19. Ho JY, Hsu RJ, Liu JM, Chen SC, Liao GS, Gao HW and Yu CP: MicroRNA-382-5p aggravates breast cancer progression by regulating the RERG/Ras/ERK signaling axis. Oncotarget 8: 22443-22459, 2017.

20. Chen C, Ridzon DA, Broomer AJ, Zhou Z, Lee DH, Nguyen JT, Barbisin M, Xu NL, Mahuvakar VR, Andersen MR, et al: Real-time quantification of microRNAs by stem-loop RT-PCR. Nucleic Acids Res 33: e179, 2005.

21. Livak KJ and Schmittgen TD: Analysis of relative gene expression data using real-time quantitative PCR and the 2(-Delta Delta C(T)) method. Methods 25: 402-408, 2001

22. Kasinski AL and Slack FJ: miRNA-34 prevents cancer initiation and progression in a therapeutically resistant K-ras and p53-induced mouse model of lung adenocarcinoma. Cancer Res 72: 5576-5587, 2012.

23. Tung SL, Huang WC, Hsu FC, Yang ZP, Jang TH, Chang JW, Chuang CM, Lai CR and Wang LH: miRNA-34c-5p inhibits amphiregulin-induced ovarian cancer stemness and drug resistance via downregulation of the AREG-EGFR-ERK pathway. Oncogenesis 6: e326, 2017.

24. Kong W, He L, Richards EJ, Challa S, Xu CX, Permuth-Wey J, Lancaster JM, Coppola D, Sellers TA, Djeu JY and Cheng JQ: Upregulation of miRNA-155 promotes tumour angiogenesis by targeting VHL and is associated with poor prognosis and triple-negative breast cancer. Oncogene 33: 679-689, 2014.

25. Allen KE and Weiss GJ: Resistance may not be futile: microRNA biomarkers for chemoresistance and potential therapeutics. Mol Cancer Ther 9: 3126-3136, 2010.

26. Zhu L, Xue F, Xu X, Xu J, Hu S, Liu S, Cui Y and Gao C: MicroRNA-198 inhibition of HGF/c-MET signaling pathway overcomes resistance to radiotherapy and induces apoptosis in human non-small-cell lung cancer. J Cell Biochem 119 7873-7886, 2018.
27. Iorio MV, Ferracin M, Liu CG, Veronese A, Spizzo R, Sabbioni S, Magri E, Pedriali M, Fabbri M, Campiglio M, et al: MicroRNA gene expression deregulation in human breast cancer. Cancer Res 65: 7065-7070, 2005

28. Blenkiron C, Goldstein LD, Thorne NP, Spiteri I, Chin SF, Dunning MJ, Barbosa-Morais NL, Teschendorff AE, Green AR, Ellis IO, et al: MicroRNA expression profiling of human breast cancer identifies new markers of tumor subtype. Genome Biol 8: R214, 2007.

29. Volinia S, Galasso M, Sana ME, Wise TF, Palatini J, Huebner K and Croce CM: Breast cancer signatures for invasiveness and prognosis defined by deep sequencing of microRNA. Proc Natl Acad Sci USA 109: 3024-3029, 2012.

30. Mirzoeva OK, Das D, Heiser LM, Bhattacharya S, Siwak D, Gendelman R, Bayani N, Wang NJ, Neve RM, Guan Y, et al: Basal subtype and MAPK/ERK kinase (MEK)-phosphoinositide 3-kinase feedback signaling determine susceptibility of breast cancer cells to MEK inhibition. Cancer Res 69: 565-572, 2009.

31. Zhao W, Ma N, Wang S, Mo Y,Zhang Z, Huang G, Midorikawa K, Hiraku Y, Oikawa S, Murata M and Takeuchi K: RERG suppresses cell proliferation, migration and angiogenesis through ERK/NF- $\kappa \mathrm{B}$ signaling pathway in nasopharyngeal carcinoma. J Exp Clin Cancer Res 36: 94, 2017.

32. Wang AG, Fang W, Han YH, Cho SM, Choi JY, Lee KH, Kim WH, Kim JM, Park MG, Yu DY, et al: Expression of the RERG gene is gender-dependent in hepatocellular carcinoma and regulated by histone deacetyltransferases. J Korean Med Sci 21: 891-896, 2006.

33. Zhang Y, Ren S, Yuan F, Zhang K, Fan Y, Zheng S, Gao Z, Zhao J, Mu T, Zhao S, et al: miR-135 promotes proliferation and stemness of oesophageal squamous cell carcinoma by targeting RERG. Artif Cells Nanomed Biotechnol 46 (Suppl): 1210-1219, 2018. 